\section{Risk factors predicting development of complications in 72 dogs with oesophageal foreign bodies: a retrospective study}

\section{Bohye Shin, Ji-Houn Kang, Min-Keun Shin, Hakhyun Kim, Woon-Bum Baek, Byeong-Teck Kang, Gonhyung Kim, Dongwoo Chang, Ki-Jeong Na, Mhan-Pyo Yang}

College of Veterinary Medicine, Chungbuk National University, Cheongju, Chungbuk, Republic of Korea

\section{PURPOSE}

Complications related to oesophageal foreign bodies are common. Oesophageal perforation and laceration are the most serious complications. The aim of this study was to determine predictive risk factors implicated in complications in dogs with oesophageal foreign bodies.

\section{MATERIALS AND METHODS}

Medical records of 72 dogs diagnosed with oesophageal foreign bodies by endoscopy were reviewed. Information included age, breed, sex, location, size, type of foreign body, and impaction duration. To identify risk factors associated with the development of complications after foreign body ingestion, categorical variables were analysed using the chi-square or Fisher's exact tests and multivariate analysis, as appropriate. $P<0.05$ was considered statistically significant.

\section{RESULTS}

Complications secondary to oesophageal foreign bodies included megaoesophagus, oesophagitis, perforation, laceration, diverticulum, and stenosis. Univariate analysis revealed that the location and duration of impaction after foreign body ingestion were related to an increased risk of oesophageal laceration and perforation in dogs. Multivariate analysis showed that age, impaction duration, and foreign body size were significant independent risk factors associated with the development of complications in dogs with oesophageal foreign bodies.

\section{CONCLUSION}

Age, foreign body size, and impaction time were important predictive factors of complications after foreign body ingestion in dogs. The risks of oesophageal laceration and perforation in particular may increase with a longer duration of impaction and a caudal location of oesophagus.

\section{Comparison of three mounts for processing endoscopic gastro-duodenal biopsies: a prospective study in dogs and cats}

\section{Guillaume Ruiz,2, Edouard Reyes- Gomez $^{2}$, Edward Hall', Valérie Freiche ${ }^{2}$}

1 University of Bristol, Bristol, UK

2 Ecole Nationale Vétérinaire d'Alfort, Maisons-Alfort, France

\section{INTRODUCTION}

Gastrointestinal endoscopic biopsy is commonly performed in dogs and cats and several factors have been reported to influence the quality of the biopsies and subsequent histopathological analysis (e.g., endoscopist experience, type of forceps, number of samples, pathologist interpretation criteria). Conversely, no recommendations have been published yet regarding handling of specimens from their collection to their processing in the laboratory.

Therefore, the aims of our study were to compare three techniques for mounting gastrointestinal endoscopic biopsies and to determine whether the techniques provide reliable diagnostic histopathological samples and if any one technique is of superior quality.

\section{MATERIALS AND METHODS}

Twenty-one dogs and two cats undergoing diagnostic upper gastrointestinal endoscopy were prospectively included. Biopsies were taken from three different sites in the stomach and from the duodenum, and were transported to the laboratory using three techniques: specimens mounted on a thin cucumber slice (as described in recent veterinary endoscopy textbooks) or on a moisturised synthetic foam sponge (both of them being then encased in plastic cassettes and immersed in formalin), or specimens floating free in formalin. After routine preparation of $4 \mu \mathrm{m}$ sections for microscopy, specimens prepared by the three techniques were compared on histopathological slides with regard to each specimen's depth, presence of artefacts, and a single, blinded pathologist's confidence in the diagnosis using a standardized questionnaire.

\section{RESULTS}

Twenty-three patients were included, and a total of 528 biopsies studied. Specimens on cucumber slice were wider compared to those on sponge or floating free in formalin, particularly for gastric specimens (mean width of 4.14 vs. 3.57 and 2.55 $\mathrm{mm}$, respectively). However, specimens on synthetic sponge had less artefacts compared to those on cucumber slice and those floating-free in formalin (artefacts absent in $85.7 \%$, $71.4 \%$ and $69 \%$, respectively). Pathologist's confidence in the diagnosis was also superior with the sponge technique over cucumber slice and floating-free specimens (confidence considered good in $88.1 \%, 73.8 \%$ and $64.3 \%$, respectively). 


\section{CONCLUSIONS}

The use of synthetic foam sponge as a mount for biopsies was found to be superior in this study and should be used when performing gastrointestinal endoscopy. We demonstrated an improvement in the quality of the specimens with this technique, and a better confidence in histopathological interpretation.

\section{Development of an algorithmic approach to the diagnosis of acute canine pancreatitis}

\section{Chiara Giannasi', Simon Tappin', Rob Harrand ${ }^{2}$, Agata Michalak ${ }^{2}$, Heike Aupperle ${ }^{3}$, Ursula Tress ${ }^{3}$, Christina Borschensky ${ }^{3}$, Marlies Staudacher, Mark Dunning ${ }^{5}$, Richard Mellanby ${ }^{6}$, Rob Foale ${ }^{1}$, Kevin Slater ${ }^{2}$}

\footnotetext{
1 Dick White Referrals, Station Farm, London Road, Six Mile Bottom, Cambridgeshire, UK

2 Avacta Animal Health, Unit 706, Avenue E, Thorp Arch Estate, Wetherby, UK

3 Laboklin GmbH\&Co.KG, Steubenstr. 4, 97688 Bad Kissingen, Germany

4 Dres. Staudacher, Trierer Str. 652, 52078 Aachen, Germany

5 School of Veterinary Medicine and Science, The University of Nottingham, Sutton Bonington Campus, Sutton Bonington, Leicestershire, UK

6 The Royal (Dick) School of Veterinary Studies, The University of Edinburgh, Easter Bush Campus, Midlothian, UK
}

\section{INTRODUCTION}

Acute canine pancreatitis $(\mathrm{ACP})$ requires a rapid and definitive diagnosis. However non-specific clinical signs and elevated serum markers mostly lack sensitivity or specificity. Consequently, confirmed diagnosis requires results from a range of tests including pancreatic imaging, serum biochemistry and physical examination. Performance of individual low specificity markers can be improved by combining multiple markers using analytical algorithms. The aim of this study was to develop and test an algorithm for use in the diagnosis ACP.

\section{MATERIALS AND METHODS}

The activity of three non-specific biomarkers, amylase, lipase and alkaline phosphatase was determined in samples from 117 dogs with clinical signs compatible with ACP (these included vomiting, abdominal pain, anorexia), formed from two groups. In group 1 (76 dogs) the possible presence of ACP was scored clinically by three blinded experienced clinicians according to the system described by McCord et al (JVIM 2012;26:888-896). Each case was independently scored after reviewing the history, results of routine haematology, serum biochemistry, cPLi assay, abdominal ultrasound, further diagnostic procedures, outcome and the final diagnosis recorded. In group 2 (41 dogs) the presence of ACP was assessed by histopathological assessment of pancreatic samples obtained during elective explorative surgery. Serum samples were obtained on admission in group 1 and within 24 hours of surgery in group 2 .

A diagnostic algorithm was developed and validated using logistic regression and a 10 fold cross validation method. This approach fits an equation linking the categorical outcome (ACP 'present' or 'absent') to the predictor variables (the biomarkers). Validation was performed by dividing the data into 10 approximately equal sized, balanced groups. One group was then used to validate a model trained on the remaining nine groups. This was repeated 10 times so that each group was used as the validation set in turn. Final performance of the algorithm was obtained from the mean of the 10 models. Finally, ROC analysis was used to select threshold values to optimise the sensitivity and specificity of the test.

\section{RESULTS}

Thirty-seven dogs were classified as having ACP (26 in group 1 and 11 in group 2). The results of the 10 fold cross validation of the algorithm resulted in a specificity of $80 \%$ and a sensitivity of $86 \%$.

\section{CONCLUSIONS}

Results suggest that test performance for ACP can be improved when multiple diagnostic parameters are combined using disease specific algorithms. 